\title{
Point-Driven Understanding in Engineering Lecture Comprehension
}

\author{
Leslie A. Olsen and Thomas N. Huckin
}

\begin{abstract}
Nonnative speakers have long been known to have trouble understanding academic lectures. ESP researchers and teachers agree that the problem lies mainly at the discourse level, not at the sentence level; accordingly, a body of discourse-oriented teaching materials for lecture comprehension is now on the market. Though a step in the right direction, these materials fail to do justice to the rhetorical, strategic nature of academic lectures. As our study shows, students may understand all the words of a lecture (including lexical connectives and other discourse markers) and yet fail to understand the lecturer's main points or logical argument.

Our study was an exploratory one. Fourteen NNS graduate and undergraduate students watched an authentic 16-minute videotaped lecture on a topic in mechanical engineering and then were asked to provide immediate-recall summaries, which were then analyzed in consultation with the lecturer. Although the lecture was clerly structured around several main points, most of the students failed to grasp these points. These results are discussed in terms of listening strategies: the successful students used a "point-driven" strategy while the unsuccessful ones used an "information-driven" strategy. We conclude that students should be taught how to listen to lectures in a more rhetorical, strategic way. More generally, if we are to teach students to understand and communicate more effectively, we should help them see how the organization of their discourse fits into the larger goals, agendas, and contexts in their fields.
\end{abstract}

\section{Introduction}

For most university students, understanding lectures is critical to academic success. In courses where lectures do not simply repeat the text, students who have trouble following lectures or taking proper notes may fail to absorb important course material; there is no text available for them to make up for what they miss in lectures. In the past decade or so, as increasing numbers of foreign students have come to study in American and British universities, many ESL teachers and researchers have noticed that this is a particularly serious problem for nonnative speakers (NNSs). Even NNSs with good scores on standard language proficiency examinations sometimes have severe problems understanding even well-structured and well-presented lectures. As Roni Lebauer has noted, "A problem common to many students in advanced listening comprehension and notetaking classes is that, in spite of apparent fluency, they still have difficulties understanding the points made in lectures" (Lebauer, 1984:41).

Address correspondence to: Leslie A. Olsen, Technical Communication Program, University of Michigan, Ann Arbor, MI 48109-2108, USA; or to Thomas N. Huckin, Department of English, University of Utah, Salt Lake City, UT 84112, USA. 
In the past, conventional wisdom decreed that the nonnative speaker's problems with lecture comprehension could be attributed to sentence-level linguistic inadequacies related to vocabulary, pronunciation, grammar, etc. However, research by Morrison (1974), Chaudron and Richards (1986) and others suggests that while linguistic shortcomings may contribute to incomprehension, they are certainly not the only impediments or even perhaps the most serious ones. Instead, discourse-level cues seem to be more important. Morrison, for example, attributes much of the lecture comprehension problem to students' inability to perceive discourse markers and logical relationships and connectives. Chaudron and Richards, in a controlled experiment, found that local "micro-markers" were less important to successful recall of a lecture than were global "macro-markers."

Realizing that many NNS lecture comprehension problems lie mainly at the discourse level, not at the sentence level, a number of ESL pedagogues have developed discourse-sensitive teaching approaches and materials. Lebauer, for example, encourages teachers to use pseudo-cloze lecture transcripts to help students anticipate the various discourse-level turns that a lecture might take. DeCarrico and Nattinger (1988) advocate the teaching of lexical phrases as cues to macrostructure of lectures. Textbooks on lecture comprehension such as those by Young and Fitzgerald (1982), Mason (1983), and Ruetten (1986) devote significant attention to discourse structure.

These advances in research and pedagogy are certainly a step in the right direction, but they are not enough. We have observed many students who, even though they understand the lecturer's use of discourse markers, still do not fully grasp the gist of a lecture. They understand how utterances "stick together" and they can anticipate various "moves," yet they do not understand the speaker's main points or the logical structure of his argument. The purpose of our study was to explore this phenomenon.

\section{Methodology}

Our methodology involved selecting a realistic sample lecture, showing it to both native and nonnative subjects, having them summarize the content of the lecture to a "friend," transcribing these summaries, and then analyzing the transcripts for completeness and accuracy of comprehension. Instead of controlling our variables, as for example Chaudron and Richards did, we elected to do exploratory research and try to create the most naturalistic, authentic conditions we could. The materials, subjects, and procedure we used are described in more detail below.

\section{Materials}

To provide a sample lecture for our subjects, we chose a 16-minute videotaped lecture segment on fracture mechanics from a first year graduate course in Mechanical Engineerng. It met the criteria we had established for such a sample lecture: it was authentic (was a real class lecture), had a level of 


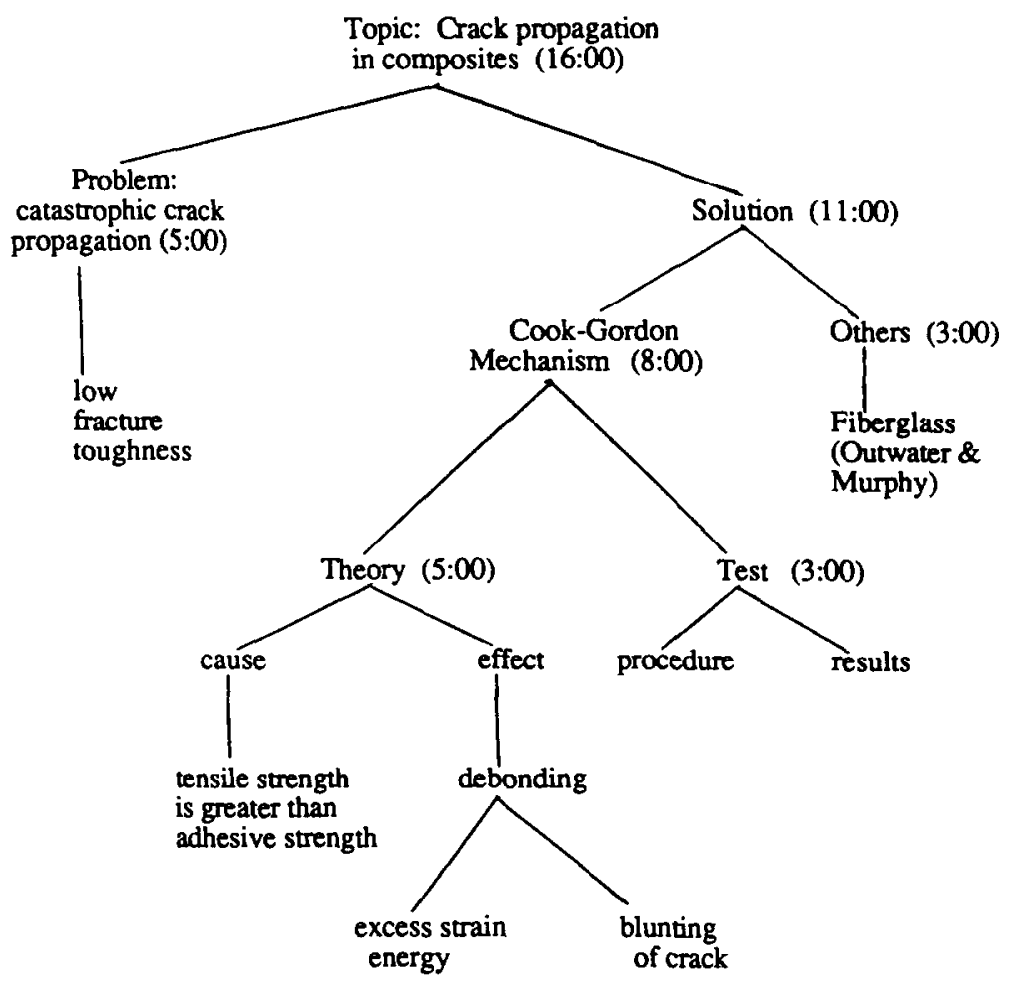

Figure 1. Overall structure of Caddell lecture, with time breakdown.

subject matter understandable to non-majors, was comprehensible out of context, and was well-organized, clear, and coherent.

As the appended material illustrates, the lecture segment describes how a crack moves through a substance and how such a crack movement (or crack propagation) can be stopped. The content of the lecture has been clearly understood by freshmen and nonengineers from a variety of backgrounds, such as political science, banking, linguistics, and education. A transcript of the first ten minutes of the segment appears in Appendix A; a tree diagram of the entire lecture appears in Figure 1. While this transcript may be somewhat difficult to understand - as is often the case with transcribed material and is especially true with engineering lectures which rely heavily on visual materials - the videotaped version of the lecture is relatively clear and easy to follow. It is an excellent example of the problem/solution focus in engineering practice and of the operation of the scientific method where the relation between theory and data is critical: theory provides a framework for the explanation of existing data and the discovery of new data, and data proves or disproves the adequacy of theory. The lecturer relied heavily on visual aids and on prosodic cues, both of which are missing from the transcript. 


\section{Subjects}

The subjects for this exploratory study were fourteen nonnative speakers of English, including ten graduate students and four undergraduates from nine different countries and eight different fields of engineering, plus physics.

\section{Procedure}

All subjects were instructed "to listen or watch for main ideas and to take notes as you would in a regular lecture situation." They were then asked "to explain what the lecturer had said as if you are telling a friend who had missed the lecture but who needed the lecture material to prepare for a test." In this task, they were allowed to use their notes and to take as much time as they needed. These immediate-recall summaries were tape-recorded, and several subjects also participated in follow-up interviews. The summaries were then transcribed and analyzed for completeness and accuracy. In doing the transcriptions, the authors created the orthographic forms of the texts presented later, marking sentence and paragraph boundaries and using a series of 3 periods (...) to indicate a pause in the oral version.

Evaluation of each subject's transcript was done qualitatively by the two authors after extensive review of the videotape and a lengthy interview and the lecturer. [Such use of a specialist or expert informant is discussed in Selinker (1979) and Huckin and Olsen (1984).] The lecturer had presented this material many times before and therefore was easily able to articulate to us the main points he wanted to get across. He told us that he wanted students to understand

a. that compositc matcrials used to be prone to severe cracking

b. that the solution to this problem lay in increasing the fracture toughness of these materials, and

c. that the Cook-Gordon theory was a major step in this direction, making people realize that there is a trade-off relationship between tensile strength and adhesive strength. More specifically, he wanted students to understand that in order to stop cracks from moving through a fiberreinforced material in a direction perpendicular to the fiber, you can create composites in which the bond between the matrix material and the fibers embedded within it is deliberately weakened. By allowing "debonding" to occur, you open up new cracks that run at right angles to the initial crack, blunting its shape and deflecting it.

Before interviewing Professor Caddell, the two authors did an independent analysis of the structure and content of the lecture. Their analysis agreed with the summary of main points later provided by Professor Caddell.

The importance of the three main points of the lecture is reflected in the tree diagram in Figure 1 which was generated by the authors in their independent analysis. In this figure the problem/solution structure is the major division 
within the lecture, the relationship of theory and test results is the main division under the Cook-Gordon mechanism, and the effect of debonding on the propagation of cracks is the most developed part of the theory section. These major points are signaled in the transcript in Appendix A, the transcript of the first ten minutes of the lecture, by such units as the following:

The real problem is that there was no way to stop the crack from propagating because the fracture toughness of the material - that composite itself - was just too low. [sentence 4]

The whole idea here when they theorize this ... [22]

Now this was a theory that was proposed, and it turmed out that experiments did tend to support it (and I'll indicate one in just a minute) [26]. But the key thing is, in this Cook and Gordon theory, it says that the debonding between the matrix and the fiber occurs before the crack reaches the fiber [27].

A "successful" summary therefore was defined as one

1. which identified the problem-solution structure of the lecture,

2. which identified the relation of theory to tests of theory, and

3. which described the effect of debonding on the propagation of cracks and the need to trade off adhesive strength for tensile strength.

\section{Results}

The results of this study were somewhat surprising. Three of our subjects failed probably due to inadequate English as evidenced by the fact that they produced short summaries (avg. 82 words) with many grammatical errors. An example of a failed summary appears in Figure 2, which is a transcription of the oral explanation by a Taiwanese aerospace engineering student who had been in the U.S.A. for 31/2 months, had studied English in Taiwan for 6 years, and had no special knowledge of the lecture subject. As mentioned earlier, in transcribing this and the following explanations, the authors created the orthographic form of the text, marking sentence and paragraph boundaries and using a series of 3 periods (...) to indicate a pause in the oral version.

\footnotetext{
First we put the force on the material. If this material hasn't is not come to ... the crack will propagate along the material, but if we have a . . material the ... then there will be another tensus stress according . . . according on the side of ... under this tensus stress, you, this tensus stress will resist the propagation along the material so if we have ... material can . . . can resist ... force than a pure material. We calculated that about $25 \%$... that's all $\ldots$
}

Figure 2. Example of failed summary probably due to inadequate English.

Note that this subject failed on all three of the criteria defined for a successful summary in this study: the subject did not

1. identify the problem-solution structure of the lecture 
2. identify the relation of theory to tests of theory or

3. describe the effect of debonding on the propagation of cracks and the need to trade off adhesive strength for tensile strength, although he was trying to deal with the effect of debonding in his summary.

Six of our subjects failed despite adequate English as indicated by the fact that they produced relatively long, fluent, and coherent summaries (averaging 349 words) and felt they had understood the lecture segment. A sample of this sort of summary appears in Figure 3 produced by a senior in Mechanical Engineering (the general area of the lecture) who had some knowledge of crack propagtion before this lecture but did not have specific knowledge of the material covered in the point of the lecture. This student has a Turkish background, had lived in the United States for about 12 years before attending the University of Michigan, and spoke Turkish at home.

\begin{abstract}
$\mathrm{OK}$ according to the lecture there is a boron material with the initial crack and has some fiber material, fiber roles inside, and has an initial crack, and load is applied to the both ends of this material, and there are more strain fractures in the material where the initial crack started and these experiments are basically done to improve the toughness of fractures in materials and one way of improving the toughness of materials or toughness of the crack is putting some other materials inside the materials that has the initial crack in it and according to the Cook-Gordon mechanism it's one theory which is talking about the material which is put improving the fracture toughness by having fiber materials inside the material fiber lines . . . but somehow there are other application that does not that does not solve the same mechanism, like having a fiber with lower sheer stress areas and higher sheer stress areas, combination of these ... and an initial crack in the material as is having a different form of fracture not exactly as the Cook-Gordon Mechanism. According to the Cook-Gordon Mechanism . . . strength has a direction opposite to the fracture propagation direction where the areas where the rnd is in the line crack area there is more bonding in the material which causes higher strength.
\end{abstract}

Question by experimenter: You are now referring to Figure A on your notes, right?

Answer by student: Yeah and Figure B which is a different structure has a fiber which...

Figure 3. Example of a failed summary due to failure to grasp the main point.

While this summary does deal somewhat with the relation of theory to tests of theory, it only indirectly identifies the problem [these experiments are basically done to improve the toughness of fractures in materials and but somehow there are other application that does not that does not solve the same mechanism], and it fails to grasp the main point (the effect of debonding on the propagation of cracks and the need to trade off adhesive strength for tensile strength).

Four of our subjects generally succeeded, and one completely succeeded, as illustrated in Figure 4, a transcription of the oral explanation of a 21-year old Chinese student in Computer Engineering, who had studied English for 14 years in his home country but had been in the United States only three months. 
Note that the student's background is in Computer Engineering while the lecture content is from Mechanical Engineering.

This naturally is about mechanical engineering and the major emphasis is on cracking in fracture and . . . for a composite material which is made of matrix and fiber cracking always occur and cracking occurs it will propagate along the . . . sorry, I mean propagate perpendicular to the matrix and fiber. This crackeling crackeling occurs and the strength and strength of the fiber-matrix decrease and also the fracture of toughness decrease. Crackeling is propagating along sorry, I mean cracking is propagated perpendicular to the mat - of matrix and fracture matrix and fiber.

I - the technical book called The New Science of Material . . . it can be - theory is being present. Consider, consider fiber, consider . . material composite of fiber-matrix and part of this material tensile stress increases and cracking occurs perpendicular to the direction of app - . . . no . . . When strength of fiber increases, debonding is occurred along - perpendicular to the fiber and strain energy is incrreased in other areas of the material; therefore, the crack, the crack is not propagated in a perp - is strictly perpendicular direction, we have to note. In fact, that there is some . . occurs in the crackin process. Some cracking occurs along the direction of the . . . while some of them occur perpendicular to the direction of note. Before the crack, before the cracks reach the fibers, bonding between fiber and matrix occur in the direction perpendicular which is in the direction parallel to the direction of note. When cracking occur there is a trade-off between strength and toughness: as strength is increased, toughness is decreased and vice versa. This process is called "Cook-Gordon mechanism" and it increased at least and this mechanism at least doubles the toughness of the composite materials. But the combination is so complex that there is no - so that there is no single theory or equation that enables us to calculate, to calculate the cracking of the composite material. Sometimes there is no debonding and sometimes . . . it doesn't and sometimes it does occur. When there is no debonding, the crack propagate directly perpendi . . . through the fibers and the fibers is not affected. There are so many variables in this kind of problem such a debonding and . . . strengthen. To this day there is no single equation or theory that enables, that enables us to . . . all possible situation . . that's all.

Figure 4. Example of successful summary.

This summary meets all three criteria for success in this study. It identifies the problem-solution structure of the lecture. It identifies the relation of theory to tests of theory [This process is called "Cook-Gordon mechanism" and it increased at least and this mechanism at least doubles the toughness of the composite materials.]. And finally, it describes the effect of debonding on the propagation of cracks and the need to trade off adhesive strength for tensile strength [When cracking occur there is a trade-off between strength and toughness: as strength is increased, toughness is decreased and vice versa.]

\section{Discussion}

The traditional teaching materials appear to attribute most of the failure in lecture comprehension to sentence-level linguistic shortcomings such as 
speech perception, vocabulary, and grammar, since these materials devote by far the majority of their attention to these issues. Even the recent textbooks by Young and Fitzgerald (1982), Mason (1983), and Ruetten (1986) heavily stress sentence-level exercises, though they also briefly discuss the main point of a lecture. This matter of emphasis may be partly attributed to the unavailability of most of the rhetorically-oriented research on lecture comprehension not already cited in this paper: much of it (by British researchers such as Candlin and Murphy, Holes, Morrison, James, and Wijasuriya) is still unpublished and thus not readily available. It may also be due to the traditional emphasis in linguistics on sentence-level phenomena and the linguistic training of many of the practitioners dealing with nonnative speakers. We note that there is a rich tradition in rhetoric - including both speech and composition that emphasizes the overall structure and goals of discourse, sometimes even to the partial exclusion of such sentence-level issues as speech perception, vocabulary, and grammar. However, linguists and rhetoricians have not traditionally had a high level of interaction.

However, the hypothesis that lecture comprehension fails because of sentence-level linguistic shortcomings does not account for our result. Six of our subjects failed despite adequate sentence-level English and their sense that they understood fully the linguistic content of the lecture. Their sentence-level English was adequate in that all of these subjects understood most of the details of the lecture, had copious and accurate notes on the details of the lecture, and produced long and fluent summaries. However, they apparently didn't see how things fit together.

Thus, factors other than narrowly linguistic ones seem to be at work here. Some of the failures missed the problem-solution rhetorical structure and the organizing role of theory in the particular problem-solving process illustrated. Both the structure and rolc of theory wcre prominently cued, as indicated by the transcript in Appendix A, and both were stressed strongly by gesture and intonation in the videotaped version of the lecture. Yet five of the six who failed were seemingly misled by narrative or chronological cues in the lecture: in the early days (sentence 1), Now, in the . . early 1960s (13), Then (23), and Now (26). The remaining failure focussed heavily on practical application, not on theory, even though much of the lecture dealt overtly with theory.

But what characterized the failures more than anything else was their inability to catch the lecturer's main points. Despite heavy cueing by the lecturer, all six of these students failed to grasp the idea that one can stop cracks from moving through a fiber-reinforced material in a direction peppendicular to the fiber by creating composites in which the bond between the matrix material and the fibers embeeded within it is deliberately weakened. Most of these six students noticed various macro-markers (cf. Chaudron and Richards) and most of them constructed a cohesive representation of the discourse. Nonetheless, they failed to grasp the speaker's main points.

Why should these types of failure occur? Typically, science and engineering students take notes by copying what is written on the blackboard; they often 
minimize the role of introductory remarks, rhetorical cues, prosodic markers, other audio-visual materials, etc. Unfortunately, it is sometimes exactly these features in American lectures which serve to outline and emphasize main points. The student who merely copies off the board catches some individual points but often does not see how they fit together. To further complicate this issue, many nonnative speakers are used to having professors in their home countries write the main points and the rhetorical structure in one corner of the board, each new point being added as it appears in the lecture. This is the equivalent of hearing "Now here comes the next main section of this lecture and it explains why the Cook-Gordon mechanism provided one type of solution to our problem of catastrophic crack propagation." Unfortuantely, for the nonnative speakers, few American professors have such obvious signposts for their lectures, even though the lectures may be well organized and carefully delivered.

On a broader level, we believe that the comprehension problems displayed by these nine students can be attributed to the use of an "information-driven" listening strategy rather than a "point-driven" one. Vipond and Hung (1984) discuss these two strategies in the context of reading, and Polanyi (1979) and others in the context of storytelling. We believe that the same distinction can be applied to lecture comprehension. Briefly, listeners using an informationdriven strategy simply try to absorb facts; they are more concerned with information per se than with the speaker's intentions or goals. By contrast, listeners using a point-driven strategy take a broader view, a more contextsensitive view of the interaction between speaker and listener, where context includes the speaker's presumed intention, the usual goals of the particular genre, the larger situation of which the discourse is a part, the potential role of relevant issues in the larger context, and even cultural effects between speakers of different cultures or subcultures.

What are the implications of a point-driven strategy? First, point-drive listeners assume that the entire discourse constitutes a pragmatic frame within which the author is constructing in Van Dijk and Kintsch's terms, a "global speech act." Thus, they try to see the discourse as having a single overriding main point and a number of subordinate points supporting it. [As outlined in the procedure section above, the main point of this lecture was that there is a trade-off relationship between tensile strength and adhesive strength (for instance, see sentence 12 in the transcript in Appendix A where "strong" means "high adhesive strength" and "improve the fracture toughness" means "improve the tensile strength"); the major subordinate points were

a. that composite materials used to be prone to severe cracking (sentences 1, 5 and 6 in the Appendix),

b. that the solution to this problem lay in increasing the fracture toughness of these materials (see sentences 4-12 - especially 4 and 12 - in Appen$\operatorname{dix} A$ ), and

c. that the Cook-Gordon theory was a major step in this direction (see sentences 13 and 17-28, especially 26).] 
Second, point-driven listeners pay close attention to discourse-level markers, intonation patterns, and other surface features - especially any unusual features - attributing deliberate authorial purpose to each. Third, pointdriven listeners construct a model of the lecturer as an intentional being, someone who is deliberately trying to make a point, not just to convey information. Of course, lectures or texts consist of points, subpoints, and information (specific facts or bits of data), but from a point-driven a speaker is conveying the facts and data of the information in an arrangement of points and subpoints whose main purpose is to "make the point" and subpoints.

Prof. Caddell's lecture was clearly point-driven. He stated in his interview with us that he wanted the lecture to make certain points (as discussed above), and he used many cues to try to achieve this purpose: e.g., macro-signals such as The real problem is (sentence 4), That's all I'm implying here (8), So I'm just indicating here (11), The whole idea here (22), and The key thing is (27); intonational emphasis (on key words and phrases like actually (19), even though (20), and debond (22); and visual cueing. Yet these cues were apparently lost on nine of our fourteen subjects.

\section{Conclusion}

The tentative conclusion to be drawn from this study, we feel, is that the teaching of macromarkers as advocted for example by Chaudron and Richards (1986) and DeCarrico and Nattringer (1988), though certainly worthwhile, is not by itself sufficient to make students aware of the discourse-level pragmatics of academic lectures. Students must also be taught to use appropriate listening strategies. If the lecturer is trying simply to convey information, then an information-driven listening strategy is appropriate. But if the lecturer is trying to build an argument - i.e., make a point and support it with various subpoints - a more context-sensitive point-driven strategy is called for. This strategy requires students to observe the lecturer's use of macromarkers, certainly, but it requires them to pay attention to other discourse-level cues as well, to draw on the larger context (e.g., the lecture as a whole, previous lectures, textbook material), and to infer the speaker's intentions and goals.

These strategies have not been adequately addressed in any published teaching materials that we are aware of, although they are receiving increased theoretical attention as indicted by Canale and Swain (1980) and Morley (forthcoming). Canale and Swain have proposed a theoretical framework for communicative competence that involves grammatical competence, sociolinguistic competence including sociocultural rules of use and rules of discourse, and strategic competence to deal with breakdowns in communication ( $p$. 29-31); and Morley has argued that one set of tasks which need to be addressed in lecture comprehension classrooms are tasks "describing and analyzing sociolinguistic dimensions including settings, roles and relationships, attitudes, topics and purposes of the communicative episode, etc." (forthcoming)

One final point should be noted about this particular lecture type. As are 
most professions, science and engineering are problem-solving professions, and science and engineering lectures often reflect this orientation. However, science and engineering students frequently do not see their fields as a series of ongoing and interrelated problems to be solved, since much of their course material consists of isolated, predefined mechanical "problems." Often students have only to figure out which formula should be used to process the data given in the problem. For instance, students in a basic course may be asked to find the pressure of gas $(\mathrm{P})$ given in a problem which also gives the gas's temperature (T), volume (V), and amount (in some form convertible to number of moles, n); of course, students know about the important formula $P V=n R T$, and thus their problem-solving becomes the mechanical process of plugging their given data into the common formula. In such situations, they mechanically solve well-defined problems using well-known tools. Thus, they rarely have to engage in the intellectual aspects of problem-solving - that is, to eliminate irrelevant data, finding missing data, reformulate a poorly formed or misleading problem statement, or define a new method to attack an uncommon or unique problem. They rarely have to deal with the problematic nature of a whole situation as a practicing scientist or engineer must. In fact, for many students, the only time they really deal with the entire intellectual problem-solving process is when and if they take a design course - usually in their senior year.

Some of the differences in intellectual investment between a more mechanical "plug in the formula" process and a more intellectual problem-solving process is illustrated in two quotes from a recent study by Herrington of written engineering discourse. This study compared results from a laboratory course (more mechanical) and a design course (more intellectually challenging) taken in the last semester of the senior year. The first quote is from a professor, the second from a typical student.

As Professor Abbott explained, "these labs have been around for so long that most of the new things are old things." In contrast, for Design, he said, "we don't know when we put these design problems together what reasonable answers are. That makes it a fun course to teach, much more fun than Lab." (Herrington, p. 344)

\footnotetext{
"Lab is a prefabricated situation and has been done 100 times and when you go in to do a Lab you just go in and say 'Okay, the professor has read 800 of these. We're just doing the same thing again. Let's just do it, write it up, and get it done ...' But, in Design, the professors are going to read 30 different designs. So the Discussion has a lot more meaning. It's your original work." (Herrington, p. 344)
}

As a result of similar observations, the college curriculum committees at several prominent engineering colleges have worked for many years to increase the amount of problem-solving in their curricula. Further, the national Accreditation Board for Engineering and Technology (ABET) now requires engineering departments to define the amount of intellectual (as opposed to mechanical) problem-solving in each course. However, this does not change the fact that many students still primarily engage in mechanical formulaplugging rather than the full intellectual process of problem-solving. 
In addition to a relative dearth of intellectual problem-solving in the curriculum, many technical texts and lectures do not stress the relationship between theory, existing data, and hypothesis-driven field testing very strongly - although the lecture chosen for this study did stress these relationships. Thus, science and engineering students often see their fields as a series of facts and situations and mathematical or experimental tools which are weakly related at best, rather than as a series of problems which, when tested and solved, open up new problems in a grand process known as the scientific method. Although it is important in science and engineering to understand the workings of the scientific method in a given field and to possess these larger problem-solving skills, these are not skills demanded often enough of students, and students often fail to have or perceive them.

Thus, a kind of disciplinary "cultural" conditioning tied to methods of science and engineering instruction seems to be largely responsible for the failure of our engineering subjects to "understand" this engineering lecture. They have simply not seen enough intellectual problem solving and its wrestling with multiple unclear phenomena, or of the operation of the scientific method with its reliance on existing data to form theory and hypotheses, which are then tested to generate new data and refine theory. Thus, our study suggests that if we are to teach students (both native and nonnative) to understand and communicate more effectively, we may need to help them see the larger goals, agendas, and contexts, in their fields as well as the organization of their discourse.

In closing, let us note that this study has focussed on a problem-solving lecture in engineering and has dealt with difficulties faced by engineering students. However, the problems noted may well be more general and afflict other disciplines as well. The humanities and social sciences are also problemsolving endeavors, though the problems they address may at times be harder to define and solve. We suspect from our contacts with both professors and students in nonengineering disciplines and from the national concern with critical thinking skills that students in nonengineering disciplines suffer from many of the limitations found in this study.

(Received September 1989)

\section{REFERENCES}

Canale, M., \& Swain, G. (1980). Theoretical bases of communicative approaches to second language teaching and testing. Applied Linguistics, 1, $1-47$.

Chaudron, C. \& Richards, J. (1986). The effect of discourse markers on the comprehension of lectures. Applied Linguistics, 7, 113-127.

DeCarrico, J. \& Nattinger, J. R. (1988). Lexical phrases for the comprehension of academic lectures. English for Specific Purposes, 7, 91-102.

Herrington, A. J. (1985). Writing in academic settings: A study of the contexts for writing in two college chemical engineering courses. Research in the Teaching of English, 19, 331-359.

Lebauer, R. (1984). Using lecture transcripts in EAP lecture comprehension 
courses. TESOL Quarterly, 8, 41-54.

Mason, A. (1983). Understanding Academic Lectures. Englewood Cliffs, NJ: Prentice-Hall.

Morrison, J. (1974). An investigation of problems in listening comprehension encountered by overseas students in the first year of postgraduate studies in science in the University of Newcastle upon Tyne. [cited in Murphy and Candlin]

Morley, J. (Forthcoming). Lecture comprehension. In M. Celce-Murcia (Ed.), Teaching English as a second or foreign language. Rowley, MA: Newbury House.

Murphy, D., \& Candlin, C. (1979). Engineering lecture discourse and listening comprehension. Practical Papers in English Language Education, 2, 1-79.

Polanyi, L. (1979). So what's the point? Semiotica, 25, 207-241.

Ruetten, M. (1986). Comprehending academic lectures. New York: Macmillan. van Dijk, T., \& Kintsch, W. (1983). Strategies of discourse comprehension. New York: Academic Press.

Vipond, D., \& Hung, R. (1984). Point-driven understanding: Pragmatic and cognitive dimensions of literary reading. Poetics, 13, 261-277.

Young, L., \& Fitzgerald, B. (1982). Listening and learning lectures. Rowley, MA: Newbury House.

\section{APPENDIX \\ Professor Robert Caddell on Fracture Mechanics: First Ten Minutes of Lecture}

I indicated, I think, in early comments I made about Chapter 9, that probably the biggest single failure in the early days of composite work was the fact that if a crack started propagating it generally went catastrophically [1]. For two reasons, really: One, although it's fundamental, can be overcome, and that is that many of the matrix-fiber combinations were made of materials themselves that were fairly brittle - their strain-to-fracture, regardless of whether they were fiber or matrix, was low [2]. But that has been overcome, and we'll talk about it in a qualitative way in just a second [3].

The real problem is that there was no way to stop the crack from propagating because the fracture toughness of the material - that composite itself - was just too low [4].

Now, in Figure 9-10, this is meant to illustrate - we used to run a little experiment in one of our courses where we took a single fiber of boron in an epoxy matrix and tried to get as good a bond along this interface as we possible could, hopefully making that as strong as possible [5]. And inevitably, when those composites were loaded and they broke, usually the fiber cracked first - because its strain-to-fracture was smaller [6] - That immediately tended to reduce, to some extent, the load-carrying capacity (because now you've lost some of the stronger material as far as your cross-sectional area goes) and the crack would just catastrophically propagate: we couldn't stop it at all [7]. That's all I'm implying here, that although in general - because of its larger sizes - I don't mean that the crack here has come about due to loading [8]. If it were just pure loading, very often the first thing the crack is going to be a fiber because its fracture strain is lower than a matrix [9]. But if a crack exists in a composite, it's more likely, on the average, to exist in the matrix because it's got a much greater area in 
which initial cracks could exist [10]. So I'm just indicating here, suppose we did have a crack here, if this is a material of very low fracture toughness, the crack starts to propagate, we're almost getting back, you might say, to a Griffth-type situation, where the stress is maintained, as the crack length increases the stress required to cause continuing propagation really decreases, if you don't start dropping the loads off it's going to go catastrophically [11]. And this is what happened with many many composites where the major factor was to try to make them as strong as they possibly could, and people began to realize, well, we've got to do something to improve the fracture toughness [12].

Now, in the - I think it was the early 1960s, these two men named Cook and Gordon - uh, this book by Gordon, by the way, if you ever want to read a technical book that almost reads like a novel, I would highly recommend it, it's uh - I always hate to push stuff like this, because you might think I have an interest in this book company, but it's a British company, it's called The New Science of Strong Materials, or Why You Don't Fall Through the Floor [13]. So I think you can even tell from the title, it's kind of - it's a terrific book, really, I think you'd very much enjoy reading it [14]. Paperback [15]. But in this, uh, he talks about the use of different type materials, and one chapter on composites he goes into this discussion - which I better draw a sketch here, because it really isn't completely shown in figure 9.11 - is what I'm gonna refer to [16].

But suppose we had a crack in part, and over here we have a fiber [17]. And we're loading it on this end [18]. Now it turns out - and I can't prove this to you in two minutes here, you'd almost have to read the original paper - but making a stress analysis, Cook, I believe, probably did the analysis, found that ahead of this crack and at right angles to the applied load there actually is a tensile stress that's set up at this interface [19]. In other words, even though the tensile effect is this way, there is a tensile result that occurs at right angle to the applied load [20].

Now you'd almost have to read the original paper to see why [21]. The whole idea here when they theorize this, and uh on the basis of analysis at least, said that well you know if this crack starts to propagate in this manner, as it gets closer and closer to this fiber, if this - the tensile stress that's set up in this direction under this applied loading - if it exceeds what they refer to as the adhesive strength, that is, the strength normal to the fiber at this interface, if this stress gets large enough it may start to open up a crack along the fiber, uh, it will debond, in other words [22]. Then, and this advancing crack comes into this region, finally - and I'm gonna highly exaggerate this - this type of argument indicated that two things could happen [23]. One is that the debonding along here would open up new crack surface area; that requires energy (the energy that's stored in the body), so if you're gonna, if you can use some of this excess strain energy to cause debonding along the interface - where this crack is parallel to the applied load and that's not gonna be as dangerous to us as if it was at right angles to the load - that it is conceivable that all the excess strain energy could be used up to do this instead of having the crack continue across the section [24]. Secondly, when the advancing crack runs into this region it sort of blunts it: It causes the entire crack shape to change, and a crack with that kind of configuration would be far less serious in general than one with a very sharp notch [25].

Now this was a theory that was proposed, and it turned out that experiments did tend to support it (and I'll indicate one in just a minute) [26]. But the key think is, in this Cook and Gordon theory, it says that the debonding between the matrix and the fiber occurs before the crack reaches the fiber [27]. So keep that in mind [28].

That was the proposed mechanism [29]. It turns out it does not work for every 
combination of fiber-matrix materials [30]. It's not a universal [31] - Uh, the tensile stress conceivably occur [32]. But even under tensile stresses here, depending upon

Leslie A. Olsen, Director of the Technical Communication Program at the University of Michigan.

Thomas N. Huckin is Associate Professor of English and member of the Writing Program at the University of Utah.

Both Huckin and Olsen do research and teach courses in technical communication, discourse analysis, and stylistics, and are co-authors of Technical Writing and Professional Communication for Non-Native Speakers of English (McGraw-Hill, 1990). 